\title{
Organic Cation Rotation and Immobilization in Pure and Mixed Methylammonium Lead-Halide Perovskites
}

Oleg Selig, ${ }^{\dagger}$ Aditya Sadhanala, ${ }^{\ddagger}$ Christian Müller, ${ }^{\S}, \|$ Robert Lovrincic, ${ }^{\S, \|}$ Zhuoying Chen, ${ }^{\perp}$ Yves L. A. Rezus, ${ }^{\dagger}$ Jarvist M. Frost, $^{\#}$ Thomas L. C. Jansen, ${ }^{\nabla}$ and Artem A. Bakulin*, ${ }^{*}, \bigcirc_{0}$

${ }^{\dagger}$ FOM Institute AMOLF, Science Park 104, Amsterdam 1098 XG, The Netherlands

${ }^{\ddagger}$ Cavendish Laboratory, University of Cambridge, JJ Thomson Ave, Cambridge CB30HE, United Kingdom

${ }^{\S}$ InnovationLab, Speyerer Strasse 4, 69115 Heidelberg, Germany

"Institut für Hochfrequenztechnik, TU Braunschweig, Schleinitzstr. 22, Braunschweig, Germany

${ }^{\perp}$ LPEM-UMR 8213, ESPCI-ParisTech/CNRS/Université Pierre et Marie Curie, 10 Rue Vauquelin, 75005 Paris, France

\# Department of Chemistry, University of Bath, Claverton Down, Bath BA2 7AY, United Kingdom

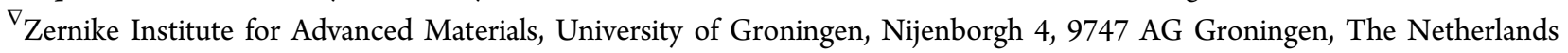

ODepartment of Chemistry, Imperial College London, London SW7 2AZ, United Kingdom

\section{Supporting Information}

ABSTRACT: Three-dimensional lead-halide perovskites have attracted a lot of attention due to their ability to combine solution processing with outstanding optoelectronic properties. Despite their soft ionic nature these materials demonstrate a surprisingly low level of electronic disorder resulting in sharp band edges and narrow distributions of the electronic energies. Understanding how structural and dynamic disorder impacts the optoelectronic properties of these perovskites is important for many applications. Here we combine ultrafast two-dimensional vibrational spectroscopy and molecular dynamics simulations to study the dynamics of the organic methylammonium (MA) cation orientation in a range of pure and mixed trihalide perovskite materials. For pure $\mathrm{MAPbX}_{3}$ $(\mathrm{X}=\mathrm{I}, \mathrm{Br}, \mathrm{Cl})$ perovskite films, we observe that the cation dynamics accelerate with decreasing size of the halide atom. This acceleration is surprising given the expected strengthening of the hydrogen bonds between the MA and the smaller halide anions, but can be explained by the increase in the polarizability with the size of halide. Much slower dynamics, up to partial immobilization of the organic cation, are observed in the mixed $\mathrm{MAPb}\left(\mathrm{Cl}_{x} \mathrm{Br}_{1-x}\right)_{3}$ and $\mathrm{MAPb}\left(\mathrm{Br}_{x} \mathrm{I}_{1-x}\right)_{3}$ alloys, which we associate with symmetry breaking within the perovskite unit cell. The observed dynamics are essential for understanding the effects of structural and dynamical disorder in perovskite-based optoelectronic systems.

\section{INTRODUCTION}

Hybrid organic-inorganic perovskite materials have recently attracted significant attention because devices made from these materials show outstanding optoelectronic properties while the fabrication procedures are themselves very simple, for example, spin coating followed by low-temperature annealing. ${ }^{1-3}$ Films of methylammonium lead iodide $\left(\mathrm{MAPbI}_{3}\right)$ are among the most investigated materials in the family of the $3 \mathrm{D}$ perovskites because they demonstrate a particularly high potential for use in thin-film solar cells with certified power conversion efficiencies exceeding $21 \%{ }^{1,4}$ At the same time, the ease with which the band gap of this compound can be tuned by merely changing the ionic composition opens up a whole new dimension for possible optoelectronic applications. ${ }^{5,6}$ The band gap can be tuned across the entire visible spectrum by varying the nature of the halide ions from $\mathrm{I}^{-}$to $\mathrm{Br}^{-}$and $\mathrm{Cl}^{-}$, which makes this
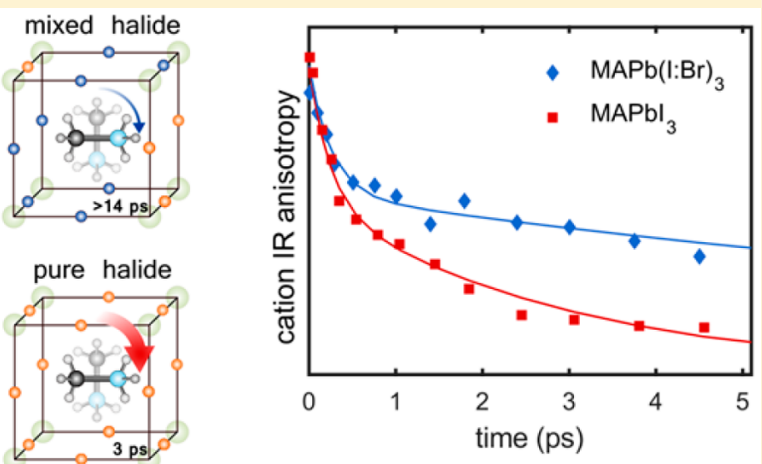

material particularly promising for tandem solar cell, light emitting diode (LEDs), and laser applications. ${ }^{5-10}$ A precise and gradual control of the band structure is achieved in the mixed halide $\mathrm{MAPb}\left(\mathrm{Cl}_{x} \mathrm{Br}_{1-x}\right)_{3}$ and $\mathrm{MAPb}\left(\mathrm{Br}_{x} \mathrm{I}_{1-x}\right)_{3}$ systems. It has been recently shown that specific combinations of ions lead to the formation of alloys with well-defined intermediate-size band gaps, surprisingly sharp band edges, and narrow distributions of emissive states. ${ }^{6,11}$

The structural properties of such "soft" hybrid perovskite materials are expected to lie at the core of their optoelectronic performance. Interionic interaction opens many degrees of freedom and allows molecular motions on multiple time and length scales. ${ }^{12}$ There are various ways in which the structural

Received: December 6, 2016

Published: February 27, 2017 
dynamics of hybrid perovskites could affect their optoelectronic properties, and to date a number of such potentially important effects have been predicted and/or observed, ${ }^{13-20}$ including band gap and exciton binding energy modulation, ${ }^{21}$ ferroelectric alignment, ${ }^{22,23}$ polaronic localization of excited states, ${ }^{14,24}$ charge transport, ${ }^{25}$ and assistance in water percolation. $^{26}$

Relatively limited information is available about the molecular-scale dynamics in these systems despite their clear importance. The motions are expected to occur on multiple time scales, and these include both ultrafast cation rotations and vibrational motions of the inorganic lattice. ${ }^{27,28}$ It is worth pointing out that these structural dynamics may be heterogeneous and may vary throughout the material depending on the microscopic composition and local structure of the perovskite. All this calls for advanced spectroscopic methods and theoretical approaches able to identify and interpret the complex variety of molecular-level processes. Substantial progress toward understanding the dynamics of $\mathrm{MAPbI}_{3}$ materials was achieved using a range of techniques ${ }^{29}$ including simulations, ${ }^{30,31}$ quasi-elastic neutron scattering, ${ }^{32-34} \mathrm{NMR}^{35}$ as well as electronic ${ }^{36}$ and vibrational ${ }^{37-39}$ spectroscopy. At the same time, less is known about the structural dynamics of the $\mathrm{Br}^{-}$and $\mathrm{Cl}^{-}$systems. ${ }^{40}$ Even fewer studies have focused on the mixed perovskites and the question as to how the structure of these materials changes as a function of their composition. ${ }^{41-43}$

Recently, we developed a method to directly track the orientational dynamics of the MA cations within the lead-halide lattice. We applied this method to $\mathrm{MAPbI}_{3}$ films and observed two characteristic time constants of motion. ${ }^{44}$ Using ab initio molecular dynamics simulations, we identified the observed dynamics as (i) fast ( 300 fs) "wobbling-in-a-cone" motions of the MA ions around the crystal axes and (ii) relatively slow $(\sim 3$ ps) jump-like reorientations of the organic ions with respect to the lead-iodide lattice. Within our $\sim 10$ ps experimental window we did not witness any long-term orientation memory nor pronounced immobilization of the MA cations. We found no evidence for the suggestion in the literature that the rearrangement of the MA would be responsible for the hysteretic behavior observed in perovskite photovoltaic devices. $^{45,46}$

Here we extend our previous approach to the chloride- and bromide-perovskites, as well as to mixed-halide systems. We observe that for pure $\mathrm{MAPbI}_{3}, \mathrm{MAPbBr}_{3}$, and $\mathrm{MAPbCl}_{3}$ films the cation dynamics accelerate with decreasing size of the halide atom and consequent shrinking unit cell of the crystal. We observe a stronger slowing, down to a partial immobilization of the cation, in the mixed $\mathrm{MAPb}\left(\mathrm{Cl}_{x} \mathrm{Br}_{1-x}\right)_{3}$ and $\mathrm{MAPb}\left(\mathrm{Br}_{x} \mathrm{I}_{1-x}\right)_{3}$ alloys. We associate these slower dynamics with a symmetry breaking in the unit cell.

We speculate that cation immobilization can affect the local dielectric environment and contribute to the increased static but decreased dynamic disorder in mixed-halide perovskite materials.

\section{RESULTS AND DISCUSSION}

Linear Infrared Spectroscopy. Figure 1 shows the IR absorption spectra of the pure and mixed perovskite films under study. Here we focus on the region $1350-1700 \mathrm{~cm}^{-1}$ where the strongest mode $\left(\sim 1475 \mathrm{~cm}^{-1}\right)$ corresponds to the $\mathrm{NH}_{3}^{+}$ bending vibrations. ${ }^{47}$ The bimodal structure of the absorption bands originates from the splitting of three degenerate vibrations into one symmetric $\left(\sim 1475 \mathrm{~cm}^{-1}\right)$ and two

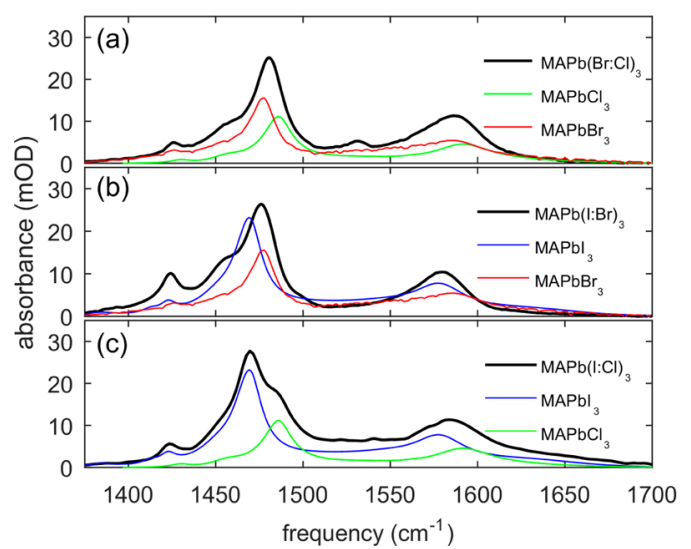

Figure 1. Linear infrared spectra of the studied materials. Every panel shows the spectrum of one hybrid sample and the corresponding pure halide spectra (a) $\mathrm{MAPb}\left(\mathrm{Cl}_{0.35} \mathrm{I}_{0.65}\right)_{3}$, (b) $\mathrm{MAPb}\left(\mathrm{Br}_{0.6} \mathrm{I}_{0.4}\right)_{3}$, and (c) $\operatorname{MAPb}\left(\mathrm{Cl}_{0.6} \mathrm{Br}_{0.4}\right)_{3}$.

antisymmetric $\left(\sim 1580 \mathrm{~cm}^{-1}\right)$ normal modes. The lowfrequency peak corresponds to the symmetric mode whose transition dipole moment is aligned with the $\mathrm{C}-\mathrm{N}$ axis of the MA molecule. Following our previous work, we use the transient anisotropy of this vibration to monitor the rotational dynamics of the organic cation. We see that the frequency of the symmetric $\mathrm{NH}_{3}{ }^{+}$-bending vibration of the MA cation decreases with increasing size of the halide anion (see Table 1).

Table 1. Resonance Frequency and Width of the $\mathrm{NH}_{3}{ }^{+}$Bend Vibration

\begin{tabular}{lcc}
\multicolumn{1}{c}{ perovskite } & frequency $\left(\mathrm{cm}^{-1}\right)$ & width $\left(\mathrm{cm}^{-1}\right)$ \\
$\mathrm{MAPbI}_{3}$ & 1468 & 21 \\
$\mathrm{MAPbBr}_{3}$ & 1476 & 17 \\
$\mathrm{MAPbCl}_{3}$ & 1486 & 18 \\
$\mathrm{MAPb}(\mathrm{I}: \mathrm{Br})_{3}$ & 1475 & 20 \\
$\mathrm{MAPb}(\mathrm{Br}: \mathrm{Cl})_{3}$ & 1479 & 20 \\
\hline
\end{tabular}

There are two effects that probably contribute to this redshift. The first effect is the decreasing strength of the $\mathrm{NH} \cdots \mathrm{X}^{-}$ hydrogen bond with increasing size of the $\mathrm{X}^{-}$anion, because bending vibrations typically show a positive correlation between hydrogen-bond strength and frequency. ${ }^{48}$ The second effect that contributes to the observed redshift is a particular localfield effect described in ref 47. Specifically, the MA vibrations redshift due to the fact that they are embedded inside and interact with a polarizable medium - the lead-halide lattice, whose polarizability increases with increasing anion size. ${ }^{47}$ The vibrational absorption spectrum of $\mathrm{MAPb}\left(\mathrm{Cl}_{x} \mathrm{I}_{1-x}\right)_{3}$ can be represented as a linear superposition of the absorption spectra of $\mathrm{MAPbI}_{3}$ and $\mathrm{MAPbCl}_{3}$. This is in agreement with previous reports, showing that $\mathrm{MAPb}\left(\mathrm{Cl}_{x} \mathrm{I}_{1-x}\right)_{3}$ does not form a homogeneous mixture but phase segregates into pure iodide and chloride phases. ${ }^{49,50}$ In contrast, the vibrational spectra of $\mathrm{MAPb}\left(\mathrm{Br}_{x} \mathrm{I}_{1-x}\right)_{3}$ and $\mathrm{MAPb}\left(\mathrm{Cl}_{x} \mathrm{Br}_{1-x}\right)_{3}$ contain a single narrow peak for each mode, positioned between the vibrational frequencies observed for the pure halide systems. This corroborates the previous X-ray diffraction and steady state optical observations ${ }^{11,6}$ that these halides are well mixed at the level of the perovskite unit cell; the MA ions experience a homogeneous and relatively narrow distribution of local environments. ${ }^{6,11}$ 


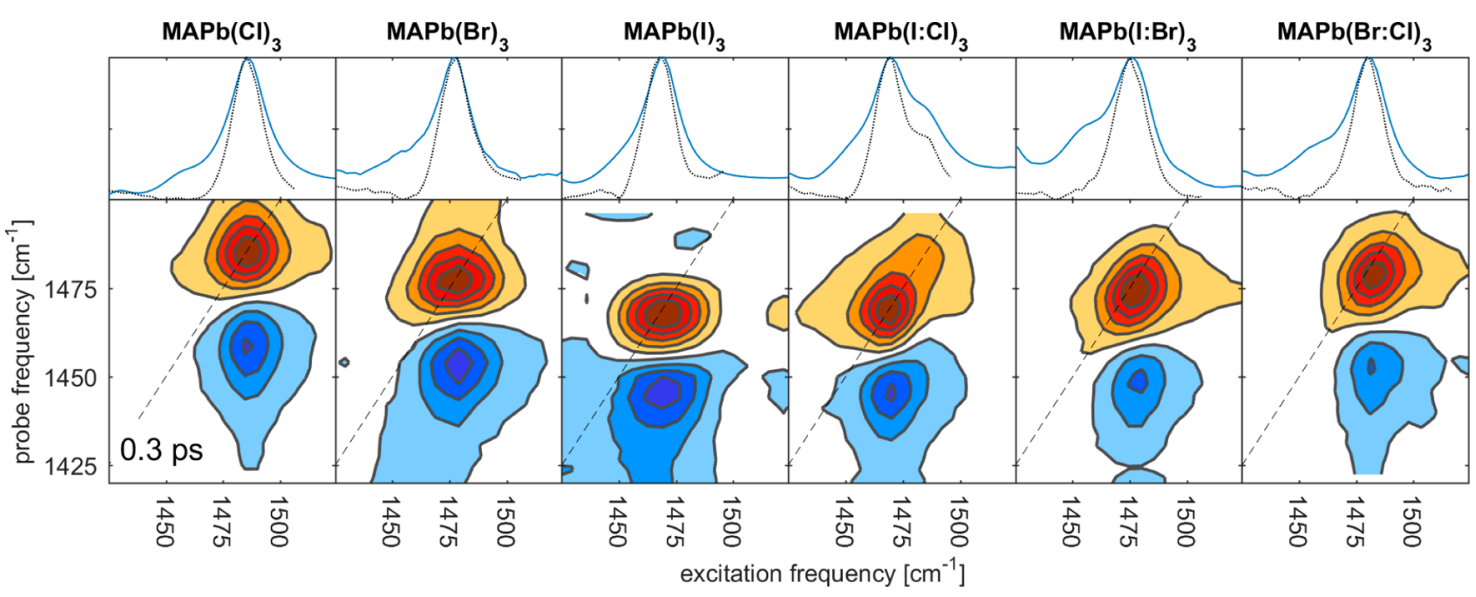

Figure 2. Bottom: Isotropic 2D spectra for the studied materials at a delay of 0.3 ps. The contour lines are placed at steps of $20 \%$ of the maximum amplitude. Red colors indicate negative absorption changes while blue colors indicate positive absorption changes. The dashed line indicates the diagonal where the pump frequency equals the probe frequency. Top: Linear infrared spectrum (blue) and slice along the diagonal of the 2DIR spectrum (black, dotted).
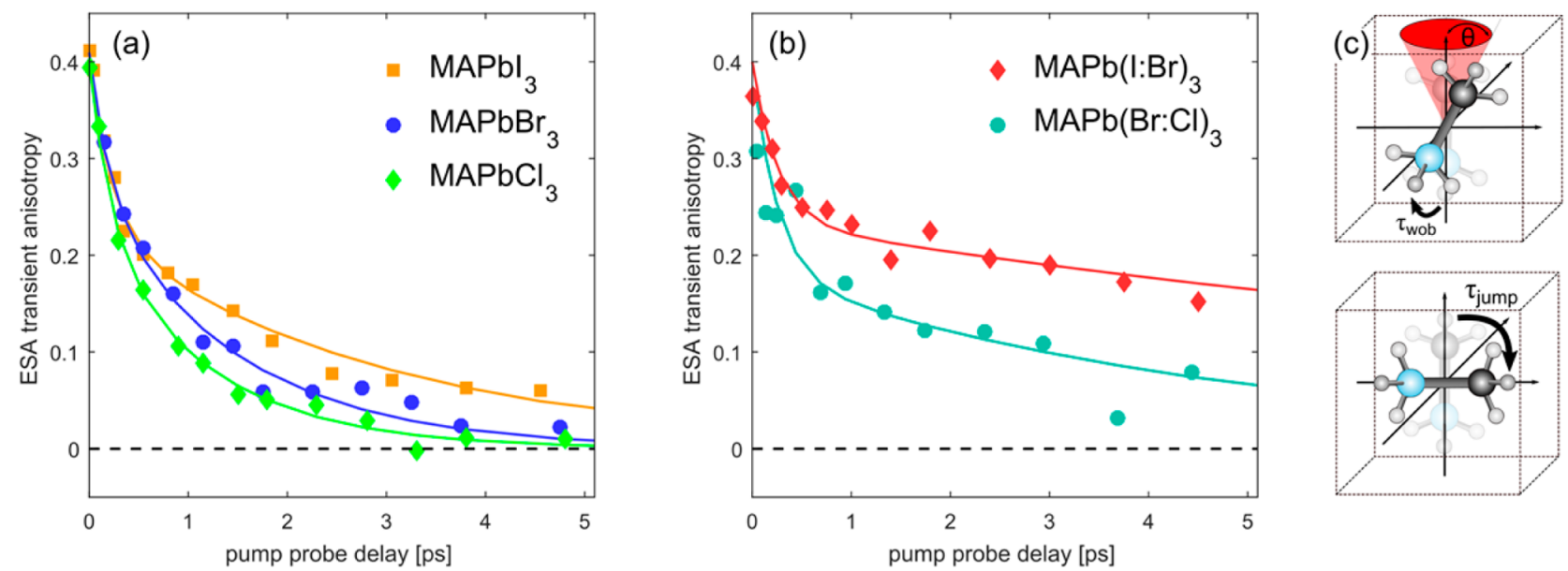

Figure 3. Transient anisotropy dynamics of the $\mathrm{MA}^{+}$ion as measured in the center of the ESA for the $\mathrm{NH}_{3}{ }^{+}$bending mode. The solid symbols show the experimentally determined anisotropy for the pure-halide perovskites in (a) and for the mixed-halide perovskites in (b). The lines show the fit with the wobbling-in-a-cone relaxation model. (c) Schematic representation of the "wobbling-in-a-cone" motion (top) and the $90^{\circ}$ angle jumps (bottom) of the $\mathrm{MA}^{+}$ion.

2DIR Spectroscopy of Pure-Halide Perovskites. To study the orientational dynamics of the organic cations, we performed 2DIR anisotropy experiments on the symmetric $\mathrm{NH}_{3}{ }^{+}$bending vibration of the MA ions. We have previously published detailed information about the experimental configuration. ${ }^{44}$ Briefly, 2DIR spectroscopy can be viewed as a version of infrared pump-probe spectroscopy, in which the transient signals are recorded as a function of both the probe and the pump frequency. This allows one to study phenomena such as mode coupling and vibrational energy transfer. ${ }^{51,52}$ In addition, the fact that the signal is spread along two frequency dimensions allows for a better separation of multiple, overlapping, resonances compared to pump-probe spectroscopy. The isotropic signal

$$
\Delta \alpha_{\text {iso }}(t)=\frac{\Delta \alpha_{\|}(t)+2 \Delta \alpha_{\perp}(t)}{3}
$$

and the anisotropy of the response

$$
r(t)=\frac{\Delta \alpha_{\|}(t)-\Delta \alpha_{\perp}(t)}{3 \Delta \alpha_{\text {iso }}(t)}
$$

can be extracted from the 2DIR measurement by acquiring the time-dependent absorption change of the probe pulse at parallel $\Delta \alpha_{\|}(t)$ and perpendicular $\Delta \alpha_{\perp}(t)$ polarization relative to the pump pulse. The former gives direct access to the relaxation dynamics, and the latter allows one to track the reorientation of the transition dipoles (and thereby the orientational motions of chemical groups). For example, the transition dipole of the symmetric $\mathrm{NH}_{3}{ }^{+}$bending vibration is aligned with the $\mathrm{C}-\mathrm{N}$ axis of the $\mathrm{MA}$ cation, so that the transient anisotropy decay of this transition follows the orientation of the cation. ${ }^{44}$

Figure 2 shows the isotropic 2DIR spectra of the studied materials. Two dominant features can be seen in every spectrum: a negative (orange) peak originating from the depletion of the vibrational ground state and stimulated emission from the first excited state; and a positive (blue) peak at a lower frequency due to excited state absorption (ESA). Interestingly, in all samples except $\mathrm{MAPb}\left(\mathrm{Cl}_{x} \mathrm{I}_{1-x}\right)_{3}$, the $2 \mathrm{D}$ lineshapes are symmetric and show no elongation along the diagonal, which points to weak inhomogeneous broadening of this vibrational mode. This lack of diagonal elongation (i.e., 
Table 2. Fitting Results of the Wobbling-in-a-Cone/Jump Relaxation Model to the Transient Anisotropy and the Scaled Correlation Function ${ }^{a}$

\begin{tabular}{|c|c|c|c|c|c|c|}
\hline \multirow[b]{2}{*}{ perovskite } & \multicolumn{2}{|c|}{$\theta(\mathrm{deg})$} & \multicolumn{2}{|c|}{$\tau_{\text {wob }}(\mathrm{ps})$} & \multicolumn{2}{|c|}{$\tau_{\text {jump }}(\mathrm{ps})$} \\
\hline & experiment & simulation & experiment & simulation & experiment & simulation \\
\hline $\mathrm{MAPbI}_{3}$ & $34 \pm 4$ & $40 \pm 1$ & $0.3 \pm 0.1$ & $0.3 \pm 0.1$ & $3.0 \pm 0.7$ & $2.6 \pm 0.3$ \\
\hline $\mathrm{MAPbBr}_{3}$ & $29 \pm 7$ & $34 \pm 1$ & $0.3 \pm 0.1$ & $0.3 \pm 0.1$ & $1.5 \pm 0.3$ & $4.1 \pm 0.3$ \\
\hline $\mathrm{MAPbCl}_{3}$ & $34 \pm 6$ & $33 \pm 1$ & $0.3 \pm 0.1$ & $0.3 \pm 0.1$ & $1.2 \pm 0.3$ & $3.8 \pm 0.3$ \\
\hline $\mathrm{MAPb}(\mathrm{I}: \mathrm{Br})_{3}$ & $34 \pm 2$ & $43 \pm 4$ & $0.3 \pm 0.1$ & $0.3 \pm 0.1$ & $15 \pm 6$ & $24 \pm 12$ \\
\hline $\mathrm{MAPb}(\mathrm{Br}: \mathrm{Cl})_{3}$ & $40 \pm 3$ & & $0.3 \pm 0.1$ & $0.3 \pm 0.1$ & $5 \pm 2$ & \\
\hline
\end{tabular}

${ }^{a}$ Simulated values give the median of fitting the scaled correlation function of $216 \mathrm{MA}$ ions and errors give the semi-interquartile range. Experimental values give the median of fitting the 2000 datasets generated by the Monte Carlo method from the errors in every data point and errors give the semi-interquartile range.
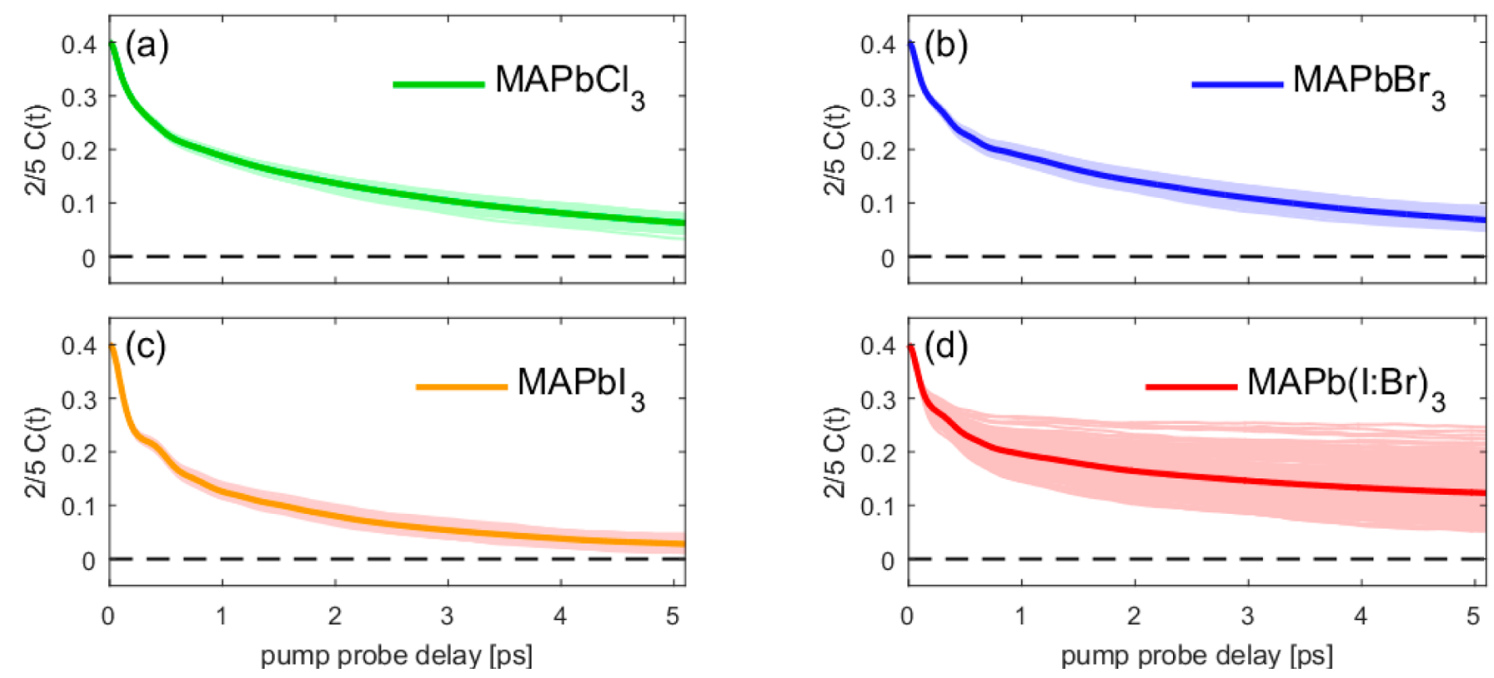

Figure 4. Rotational correlation functions of the MA ions as calculated based on classical molecular dynamics simulations for the pure-halide perovskites $\mathrm{MAPbCl}_{3}(\mathrm{a}), \mathrm{MAPbBr}_{3}(\mathrm{~b}), \mathrm{MAPbI}_{3}(\mathrm{c})$, and for the mixed-halide perovskite $\mathrm{MAPb}(\mathrm{I}: \mathrm{Br})_{3}(\mathrm{~d})$. The faint lines represent the individual correlation functions for the different MA ions in the simulation, while the thick lines represent the average over the complete calculated ensemble.

inhomogeneous broadening) suggests that the MA ions experience a narrow distribution of local environments in both $\operatorname{MAPb}\left(\mathrm{Br}_{x} \mathrm{I}_{1-x}\right)_{3}$ and $\operatorname{MAPb}\left(\mathrm{Cl}_{x} \mathrm{Br}_{1-x}\right)_{3}$. In contrast, $\mathrm{MAPb}\left(\mathrm{Cl}_{x} \mathrm{I}_{1-x}\right)_{3}$ shows a pronounced elliptical line shape which can be interpreted as a superposition of the 2DIR spectra of the chloride and iodide perovskites. We observe no sign of cross peaks between the chloride and iodide components, which confirms the presence of strong phase segregation ${ }^{50}$ as well as a lack of interaction (or interconversion) between the MA cations in the different phases.

Previously, we showed ${ }^{44}$ that the region around the groundstate bleach might be affected by a thermal response which makes it difficult to extract the molecular reorientation from this signal. Consequently, we focus on the anisotropy dynamics of the ESA region, which is not affected by the thermal signal.

Figure 3 a shows the transient anisotropy dynamics averaged over the central region of the excited state absorption (ESA) peaks for the pure-halide perovskites. In general, the dynamics involve two components similar to those which we have previously attributed to a confined wobbling $(\sim 300 \mathrm{fs})$ of the MA molecules and to jumplike reorientations between the halide unit cell facets. ${ }^{44}$ In order to extract the time constants associated with these motions we have fitted the previously used wobbling-in-a-cone relaxation model:

$$
\begin{aligned}
r(t) & =0.4 \mathrm{e}^{-t / \tau_{\text {jump }}}\left(S^{2}+\left(1-S^{2}\right) \mathrm{e}^{-t / \tau_{\text {wob }}}\right) \quad \text { with } \\
S & =\cos (\theta)(1+\cos (\theta)) / 2
\end{aligned}
$$

to the anisotropy decays. ${ }^{53,44}$ The resulting jump rates $\tau_{\text {jump, }}$, cone semiangles $\theta$, and wobbling time constants $\tau_{\text {wob }}$ are summarized in Table 2. Perhaps surprisingly, the general trend observed is that as we move to smaller halide atoms and smaller unit cell sizes (from $\mathrm{I}^{-}$to $\mathrm{Br}^{-}$and then to $\mathrm{Cl}^{-}$) the reorientation of the MA ions accelerates. This acceleration is primarily due to the increased probability for the occurrence of large-angle jumps; the fast process associated with the wobbling motion seems unaffected and can be well described by the same time constant for all five samples. This can be understood in terms of the large-angle jumps being driven by the tilting modes of the inorganic perovskite cage, whereas the wobblingmotion is a characteristic of the molecule rattling within the inorganic cage. ${ }^{54,55}$ The variation of time constant for the large scale jumps qualitatively agrees with the shift in frequency for the coupled cage-organic modes. ${ }^{56}$

Molecular Dynamics Simulations of Pure-Halide Perovskites. To support our experimental findings we have performed classical polarizable MD simulations with a $6 \times 6 \times$ 6 unit cubic super cell. The simulation details are given in the Supporting Information (SI) together with a validation against ab initio MD simulations of these perovskites using an identical simulation protocol to that used for previous $\mathrm{MAPbI}_{3}$ simulations with a $2 \times 2 \times 2$ super cell. ${ }^{57}$ Here we will focus 
on the classical results as insufficient statistics can be obtained from the ab initio simulations for the halide mixtures that we will consider later. We have used these simulations to compute the second-order correlation function of the MA orientations. This correlation function is proportional to the experimentally determined transient anisotropy and is expressed as $R(t)=2$ / $5\left\langle P_{2}(\hat{\mu}(t) \hat{\mu}(0))\right\rangle$, where $P_{2}(x)$ is the second Legendre polynomial, $\hat{\mu}(t)$ is a unit vector pointing along the $\mathrm{CN}$-axis of the MA ion, and $\langle\cdots\rangle$ denotes an ensemble average. Clearly the anisotropy curves extracted from the MD trajectories show the same two-component dynamics as the experimental anisotropies (Figure $4 a-c$ ); however, neither the classical model nor the $\mathrm{ab}$ initio simulations (presented in the SI) reproduce the experimental halide size dependence of the slow component.

At first sight, it may seem surprising that the reorientation of the MA ions speeds up along the series $\mathrm{MAPbI}_{3} \rightarrow \mathrm{MAPbBr}_{3}$ $\rightarrow \mathrm{MAPbCl}_{3}$ because, as was already mentioned above, the $\mathrm{NH} \cdots \mathrm{X}^{-}$hydrogen bond should strengthen when one moves in this direction. However, it should be realized that it is not the absolute strength of the hydrogen-bond, which determines the reorientation rate, but rather the activation barrier for moving between two equivalent hydrogen bonded orientations of the MA cation. This activation barrier is due to the fact that in the transition state the hydrogen bond is slightly elongated compared to the stable positions. Apparently, the energy penalty for lengthening the hydrogen-bond in the transition state decreases in the direction $\mathrm{MAPbI}_{3} \rightarrow \mathrm{MAPbBr}_{3} \rightarrow$ $\mathrm{MAPbCl}_{3}$. This is probably due to the fact that the unit cell size decreases for this series, which in turn causes the potential confining the MA ions to become more spherically symmetric. A similar situation is encountered in the reorientation of water where molecules engaging in so-called bifurcated hydrogen bonds represent a low-energy transition state which speeds up reorientation. ${ }^{58-60}$ We speculate that the activation energy is not well reproduced by our molecular dynamics simulations. This may not be surprising as the force field was not optimized for reproducing this. With a difference in rate in the order of a factor 2 this corresponds to a difference in the $2.5 \mathrm{~kJ} / \mathrm{mol}$ range for the activation energy.

In the picture sketched above, we have described the reorientation of the MA ion as an activated process, and we have attributed the change in reorientation rate to a change in activation barrier. However, the rate of an activated process is not only determined by the height of activation barrier but also by the dynamic changes in this barrier. Recent X-ray scattering experiments and DFT calculations suggested a moderate to strong coupling between the deformation of the inorganic lattice and the rotational motion of the organic cation. ${ }^{30,52}$ Therefore, one could imagine that the reorientation of the MA ion is facilitated by the low-frequency vibrations of the leadhalide lattice. In this scenario, the modulation of the barrier for rotational motions is directly given by the frequency of these lattice vibrations. Recent infrared and Raman experiments have shown that the frequency of these lattice modes typically increases along the series $\mathrm{MAPbI}_{3} \rightarrow \mathrm{MAPbBr}_{3} \rightarrow$ $\mathrm{MAPbCl}_{3}, 25,41,56$ and this trend would be in line with the observed increased in the MA reorientation rate. Summarizing, according to this interpretation the speeding up of the MA reorientation with decreasing size of the halide anion is attributed to two effects that work in the same direction: an increasing influence of lead-halide lattice vibrations and a decreasing activation barrier.
2DIR Spectroscopy of Mixed-Halide Perovskites. Next, we turn to the mixed-halide perovskites. Figure $3 \mathrm{~b}$ presents the transient anisotropy recorded at the center of the ESA peaks for the $\mathrm{MAPb}\left(\mathrm{Br}_{x} \mathrm{I}_{1-x}\right)_{3}$ and $\mathrm{MAPb}\left(\mathrm{Cl}_{x} \mathrm{Br}_{1-\mathrm{x}}\right)_{3}$ alloys. We do not discuss the anisotropy of the $\operatorname{MAPb}\left(\mathrm{Cl}_{x} \mathrm{I}_{1-x}\right)_{3}$ system as it represents a trivial superposition of the chloride and iodide components (see the SI). The short-time dynamics in $\mathrm{MAPb}\left(\mathrm{Br}_{x} \mathrm{I}_{1-\mathrm{x}}\right)_{3}$ and $\mathrm{MAPb}\left(\mathrm{Cl}_{x} \mathrm{Br}_{1-\mathrm{x}}\right)_{3}$ are similar to those in the other pure- and mixed-halide systems, which indicates that the wobbling motions are well activated in all these materials. However, at longer time delays significantly slower anisotropy decays are observed for the mixed-halide perovskites, compared to the pure-halide perovskites. This slowing down indicates that the molecular jumps between different unit cell facets are strongly suppressed in the mixed-halide materials and that each individual MA cation remains predominantly oriented in a particular direction.

This immobilization can be understood by considering the activation energy for the rotational motion (similarly to the pure-halide systems). For the mixed-halide systems, the halide lattice site is occupied by a statistical mixture of two halide ions. As a consequence the different MA orientations will no longer be characterized by identical hydrogen-bond strengths (as was the case for the pure-halide systems). Instead the statistical, usually asymmetric, distribution of the halide ions will lead to the presence of specific MA orientations which have an energetic minimum in their rotational potential. For these MA orientations, the activation energy for a $90^{\circ}$ jump will obviously be much larger compared to the case of the pure-halide perovskites, for which reorientation between stable positions is not associated with a change in hydrogen-bonding energy. This means that the random substitution of halogen sites within the inorganic cage effectively generates an asymmetric pocket which pins down the orientation of the MA ions.

Molecular Dynamics Simulations of Mixed-Halide Perovskites. The mixed perovskites were modeled using classical polarizable molecular dynamics simulations as described in the SI. This allowed using $6 \times 6 \times 6$ supercells with 216 organic cations to obtain sufficient sampling of the different halogen environments. The halogen atoms were inserted in a statistical manner resulting in a heterogeneous distribution of unit cells with $x=0.6$. Organic cations with anywhere from 3 to $11 \mathrm{Br}^{-}$ions in the first coordination sphere were found. Figure $4 \mathrm{~d}$ presents the rotational correlation function for a set of individual MA cations (in the mixed $\mathrm{MAPb}\left(\mathrm{Br}_{x} \mathrm{I}_{1-x}\right)_{3}$ perovskite) as well as the overall ensemble average. As clearly seen from the individual pink transients the distribution of correlation functions is very broad for the mixedhalide systems, in contrast to the narrow distribution for individual cations in the pure-halide perovskites (Figure $4 a-c$ ). As a general trend the jump time was found to be slower for cations surrounded by an equal number of $\mathrm{Br}^{-}$and $\mathrm{I}^{-}$ions than for cations in a more homogeneous environment. Considering the number of possible ways to construct these unit cells and the fact that every cation can form three hydrogen bonds, a complete analysis of the factors determining the slowdown was not possible. However, we can generally state that altering the symmetry of the unit cell should provide anisotropy and the preferred direction for the cation orientation. Changing the orientation in such way that the number of hydrogen bonds to a particular type of halogen is preserved should be more favorable as compared to when the type of halogen is changing. The correlation between the number of bromine atoms 
surrounding the MA cation and the heterogeneity of the slow rotational is given in Figures S5 and S6 (SI) along with illustrations of halogen configurations resulting in fast and slow dynamics. Interestingly, recent crystallographic investigations ${ }^{61}$ have shown that phase transition in MAPbI3 significantly alters the dynamic behavior of $\mathrm{MA}^{+}$. At room temperature, MAPbI3 adopts the tetragonal phases, unlike all other pure-halide systems studied in this work. However, we observe a clear trend in the orientation dynamics indicating that interaction with the halide atoms is more important for MA motion than the geometry of unit cell.

Interestingly, the observed immobilization of the organic cation in mixed-halide perovskites correlates with a number of trends previously observed in material properties. For example, previous studies indicated that both the emissive bandwidth and the Urbach energy are persistently smaller in pure-halide systems compared to their respective mixtures. ${ }^{6,11}$ Similar trends are observed in the structural measurements like XRD and in the charge transporting properties. ${ }^{43}$ All these observations point to an increased level of disorder in mixedhalide systems compared to pure-halide systems. Our new results indicate that, at least as far as the organic cation is concerned, the static and dynamic disorders change differently upon mixing different halide ions in the material. The static disorder is clearly increased in mixed halide systems as observed in the elongation of $2 \mathrm{D}$ peaks in Figure $2 .^{62}$ At the same time, the dynamic motions are clearly suppressed by breaking the symmetry of the unit cell. With suppressed dynamics, the static dielectric constant will decrease (the molecules can no longer respond) potentially explaining the higher exciton binding energy reported for mixed $\operatorname{MAPb}\left(\mathrm{Br}_{x} \mathrm{I}_{1-x}\right)_{3}$ perovskites. $^{63}$ While we do not have direct evidence to associate the change in electronic properties with the variations in the cation dynamics, we believe that the observed correlation manifests a clear link between electronic and structural dynamics in perovskite materials.

\section{CONCLUSION}

In conclusion, we presented a $2 \mathrm{D}$ IR and MD simulation study of the orientational dynamics of the organic cation in a range of pure- and mixed-halide perovskite materials. In the mixed $\operatorname{MAPb}\left(\mathrm{Cl}_{x} \mathrm{Br}_{1-x}\right)_{3}$ and $\operatorname{MAPb}\left(\mathrm{Br}_{x} \mathrm{I}_{1-x}\right)_{3}$ alloys we observed a higher degree of static disorder and much slower structural dynamics down to partial immobilization of the organic cations. We associate these effects with the symmetry breaking within the halide unit cell. The observed effects of the halide composition on the structural and dynamical properties of perovskite materials may be partly responsible for the previously observed variations in their optical and electronic properties.

\section{ASSOCIATED CONTENT}

\section{S Supporting Information}

The Supporting Information is available free of charge on the ACS Publications website at DOI: 10.1021/jacs.6b12239.

Sample preparation and characterization, linear FTIR spectra deconvolution, 2D spectra and different delay times and population dynamics, details of molecular dynamics simulations, analysis of molecular dynamics in mixed perovskite systems (PDF)

\section{AUTHOR INFORMATION}

\section{Corresponding Author}

*a.bakulin@imperial.ac.uk

ORCID

Artem A. Bakulin: 0000-0002-3998-2000

Notes

The authors declare no competing financial interest.

\section{ACKNOWLEDGMENTS}

We thank Maxim Pschenichnikov for useful discussions and Tobias Glaser for providing the linear IR absorption spectra. This work was supported by The Netherlands Organization for Scientific Research (NWO) through the "Stichting voor Fundamenteel Onderzoek der Materie" (FOM) research program. A.A.B. is currently a Royal Society University Research Fellow. Z.C. acknowledge the ANR-2011-JS09-00401-PvCoNano project and the EU Marie Curie Career Integration Grant (303824). A.A.B. and Z.C. thank DutchFrench Academy for the support through van Gogh grant. A.S. acknowledges the support from the India-UK APEX project. J.M.F. was supported by the EPSRC (EP/K016288/1), with computing time through the Materials Chemistry Consortium on the ARCHER facility (EP/L000202).

\section{REFERENCES}

(1) Lee, M. M.; Teuscher, J.; Miyasaka, T.; Murakami, T. N.; Snaith, H. J. Science 2012, 338, 643.

(2) Brenner, T. M.; Egger, D. A.; Kronik, L.; Hodes, G.; Cahen, D. Nat. Rev. Mater. 2016, 1, 15007.

(3) Manser, J. S.; Christians, J. A.; Kamat, P. V. Chem. Rev. 2016, 116, 12956

(4) Saliba, M.; Matsui, T.; Seo, J.-Y.; Domanski, K.; Correa-Baena, J.P.; Nazeeruddin, M. K.; Zakeeruddin, S. M.; Tress, W.; Abate, A.; Hagfeldt, A.; Gratzel, M. Energy Environ. Sci. 2016, 9, 1989.

(5) Noh, J. H.; Im, S. H.; Heo, J. H.; Mandal, T. N.; Seok, S. I. Nano Lett. 2013, 13, 1764.

(6) Sadhanala, A.; Ahmad, S.; Zhao, B.; Giesbrecht, N.; Pearce, P. M.; Deschler, F.; Hoye, R. L. Z.; Gödel, K. C.; Bein, T.; Docampo, P.; Dutton, S. E.; De Volder, M. F. L.; Friend, R. H. Nano Lett. 2015, 15, 6095.

(7) Tan, Z.-K.; Moghaddam, R. S.; Lai, M. L.; Docampo, P.; Higler, R.; Deschler, F.; Price, M.; Sadhanala, A.; Pazos, L. M.; Credgington, D.; Hanusch, F.; Bein, T.; Snaith, H. J.; Friend, R. H. Nat. Nanotechnol. 2014, 9, 687.

(8) Xing, G.; Mathews, N.; Lim, S. S.; Yantara, N.; Liu, X.; Sabba, D.; Grätzel, M.; Mhaisalkar, S.; Sum, T. C. Nat. Mater. 2014, 13, 476.

(9) Deschler, F.; Price, M.; Pathak, S.; Klintberg, L. E.; Jarausch, D.D.; Higler, R.; Hüttner, S.; Leijtens, T.; Stranks, S. D.; Snaith, H. J.; Atatüre, M.; Phillips, R. T.; Friend, R. H. J. Phys. Chem. Lett. 2014, 5, 1421.

(10) McMeekin, D. P.; Sadoughi, G.; Rehman, W.; Eperon, G. E.; Saliba, M.; Hörantner, M. T.; Haghighirad, A.; Sakai, N.; Korte, L.; Rech, B.; Johnston, M. B.; Herz, L. M.; Snaith, H. J. Science 2016, 351, 151.

(11) Sadhanala, A.; Deschler, F.; Thomas, T. H.; Dutton, S. E.; Goedel, K. C.; Hanusch, F. C.; Lai, M. L.; Steiner, U.; Bein, T.; Docampo, P.; Cahen, D.; Friend, R. H. J. Phys. Chem. Lett. 2014, 5, 2501.

(12) Egger, D. A.; Rappe, A. M.; Kronik, L. Acc. Chem. Res. 2016, 49, 573.

(13) Mosconi, E.; De Angelis, F. ACS Energy Lett. 2016, 1, 182.

(14) Long, R.; Liu, J.; Prezhdo, O. V. J. Am. Chem. Soc. 2016, 138, 3884. 
(15) Chang, A. Y.; Cho, Y.-J.; Chen, K.-C.; Chen, C.-W.; Kinaci, A.; Diroll, B. T.; Wagner, M. J.; Chan, M. K. Y.; Lin, H.-W.; Schaller, R. D. Adv. Energy Mater. 2016, 6, 1600422.

(16) Gélvez-Rueda, M. C.; Cao, D. H.; Patwardhan, S.; Renaud, N.; Stoumpos, C. C.; Schatz, G. C.; Hupp, J. T.; Farha, O. K.; Savenije, T. J.; Kanatzidis, M. G.; Grozema, F. C. J. Phys. Chem. C 2016, 120, 16577.

(17) Zhou, Y.; You, L.; Wang, S.; Ku, Z.; Fan, H.; Schmidt, D.; Rusydi, A.; Chang, L.; Wang, L.; Ren, P.; Chen, L.; Yuan, G.; Chen, L.; Wang, J. Nat. Commun. 2016, 7, 11193.

(18) Berdiyorov, G. R.; Kachmar, A.; El-Mellouhi, F.; Carignano, M. A.; El-Amine Madjet, M. J. Phys. Chem. C 2016, 120, 16259.

(19) Wright, A. D.; Verdi, C.; Milot, R. L.; Eperon, G. E.; PerezOsorio, M. A.; Snaith, H. J.; Giustino, F.; Johnston, M. B.; Herz, L. M. Nat. Commun. 2016, 7, 11755 .

(20) Zhu, H.; Miyata, K.; Fu, Y.; Wang, J.; Joshi, P. P.; Niesner, D.; Williams, K. W.; Jin, S.; Zhu, X.-Y. Science 2016, 353, 1409.

(21) Motta, C.; Mandal, P.; Sanvito, S. Phys. Rev. B: Condens. Matter Mater. Phys. 2016, 94, 045202.

(22) Pecchia, A.; Gentilini, D.; Rossi, D.; Auf der Maur, M.; Di Carlo, A. Nano Lett. 2016, 16, 988.

(23) Deretzis, I.; Di Mauro, B. N.; Alberti, A.; Pellegrino, G.; Smecca, E.; La Magna, A. Sci. Rep. 2016, 6, 24443.

(24) Zhu, X. Y.; Podzorov, V. J. Phys. Chem. Lett. 2015, 6, 4758.

(25) Sendner, M.; Nayak, P. K.; Egger, D. A.; Beck, S.; Müller, C.; Epding, B.; Kowalsky, W.; Kronik, L.; Snaith, H. J.; Pucci, A.; Lovrincic, R. Mater. Horiz. 2016, 3, 613.

(26) Müller, C.; Glaser, T.; Plogmeyer, M.; Sendner, M.; Döring, S.; Bakulin, A. A.; Brzuska, C.; Scheer, R.; Pshenichnikov, M. S.; Kowalsky, W.; Pucci, A.; Lovrinčić, R. Chem. Mater. 2015, 27, 7835.

(27) Motta, C.; El-Mellouhi, F.; Kais, S.; Tabet, N.; Alharbi, F.; Sanvito, S. Nat. Commun. 2015, 6, 7026.

(28) Deng, X.; Wen, X.; Huang, S.; Sheng, R.; Harada, T.; Kee, T. W.; Green, M.; Ho-Baillie, A. J. Phys. Chem. C 2016, 120, 2542.

(29) Herz, L. M. Annu. Rev. Phys. Chem. 2016, 67, 65.

(30) Carignano, M. A.; Saeed, Y.; Aravindh, S. A.; Roqan, I. S.; Even,

J.; Katan, C. Phys. Chem. Chem. Phys. 2016, 18, 27109.

(31) Bechtel, J. S.; Seshadri, R; Van der Ven, A. J. Phys. Chem. C 2016, 120, 12403.

(32) Leguy, A. M. A.; Frost, J. M.; McMahon, A. P.; Sakai, V. G.; Kochelmann, W.; Law, C.; Li, X.; Foglia, F.; Walsh, A.; O’Regan, B. C.; Nelson, J.; Cabral, J. T.; Barnes, P. R. F. Nat. Commun. 2015, 6, 7124.

(33) Chen, T.; Foley, B. J.; Ipek, B.; Tyagi, M.; Copley, J. R. D.; Brown, C. M.; Choi, J. J.; Lee, S.-H. Phys. Chem. Chem. Phys. 2015, 17, 31278 .

(34) Page, K.; Siewenie, J. E.; Quadrelli, P.; Malavasi, L. Angew. Chem., Int. Ed. 2016, 55, 14320.

(35) Wasylishen, R. E.; Knop, O.; Macdonald, J. B. Solid State Commun. 1985, 56, 581.

(36) Fabini, D. H.; Hogan, T.; Evans, H. A.; Stoumpos, C. C.; Kanatzidis, M. G.; Seshadri, R. J. Phys. Chem. Lett. 2016, 7, 376.

(37) Xie, L.-Q.; Zhang, T.-Y.; Chen, L.; Guo, N.; Wang, Y.; Liu, G.K.; Wang, J.-R.; Zhou, J.-Z.; Yan, J.-W.; Zhao, Y.-X.; Mao, B.-W.; Tian, Z.-Q. Phys. Chem. Chem. Phys. 2016, 18, 18112.

(38) Idigoras, J.; Todinova, A.; Sanchez-Valencia, J. R.; Barranco, A.; Borras, A.; Anta, J. A. Phys. Chem. Chem. Phys. 2016, 18, 13583.

(39) Ledinský, M.; Löper, P.; Niesen, B.; Holovský, J.; Moon, S.-J.; Yum, J.-H.; De Wolf, S.; Fejfar, A.; Ballif, C. J. Phys. Chem. Lett. 2015, 6, 401 .

(40) Motta, C.; El-Mellouhi, F.; Sanvito, S. Phys. Rev. B: Condens. Matter Mater. Phys. 2016, 93, 235412.

(41) Niemann, R. G.; Kontos, A. G.; Palles, D.; Kamitsos, E. I.; Kaltzoglou, A.; Brivio, F.; Falaras, P.; Cameron, P. J. J. Phys. Chem. C 2016, 120, 2509.

(42) Brivio, F.; Caetano, C.; Walsh, A. J. Phys. Chem. Lett. 2016, 7, 1083.

(43) Rehman, W.; Milot, R. L.; Eperon, G. E.; Wehrenfennig, C.; Boland, J. L.; Snaith, H. J.; Johnston, M. B.; Herz, L. M. Adv. Mater. 2015, 27, 7938.
(44) Bakulin, A. A.; Selig, O.; Bakker, H. J.; Rezus, Y. L. A.; Müller, C.; Glaser, T.; Lovrincic, R.; Sun, Z.; Chen, Z.; Walsh, A.; Frost, J. M.; Jansen, T. L. C. J. Phys. Chem. Lett. 2015, 6, 3663.

(45) Fan, Z.; Xiao, J.; Sun, K.; Chen, L.; Hu, Y.; Ouyang, J.; Ong, K. P.; Zeng, K.; Wang, J. J. Phys. Chem. Lett. 2015, 6, 1155.

(46) Frost, J. M.; Butler, K. T.; Walsh, A. APL Mater. 2014, 2, 081506.

(47) Glaser, T.; Müller, C.; Sendner, M.; Krekeler, C.; Semonin, O. E.; Hull, T. D.; Yaffe, O.; Owen, J. S.; Kowalsky, W.; Pucci, A.; Lovrincic, R. J. Phys. Chem. Lett. 2015, 6, 2913.

(48) Van Thiel, M.; Becker, E. D.; Pimentel, G. C. J. Chem. Phys. 1957, 27, 486.

(49) Nie, W.; Tsai, H.; Asadpour, R.; Blancon, J.-C.; Neukirch, A. J.; Gupta, G.; Crochet, J. J.; Chhowalla, M.; Tretiak, S.; Alam, M. A.; Wang, H.-L.; Mohite, A. D. Science 2015, 347, 522.

(50) Pistor, P.; Borchert, J.; Fränzel, W.; Csuk, R.; Scheer, R. J. Phys. Chem. Lett. 2014, 5, 3308.

(51) Hamm, P.; Lim, M. H.; Hochstrasser, R. M. J. Phys. Chem. B 1998, 102, 6123.

(52) Bakulin, A. A.; Cringus, D.; Pieniazek, P. A.; Skinner, J. L.; Jansen, T. L.; Pshenichnikov, M. S. J. Phys. Chem. B 2013, 117, 15545.

(53) Ji, M.; Gaffney, K. J. J. Chem. Phys. 2011, 134, 044516.

(54) Comin, R.; Crawford, M. K.; Said, A. H.; Herron, N.; Guise, W. E.; Wang, X.; Whitfield, P. S.; Jain, A.; Gong, X.; McGaughey, A. J. H.; Sargent, E. H. Phys. Rev. B: Condens. Matter Mater. Phys. 2016, 94, 094301.

(55) Beecher, A. N.; Semonin, O. E.; Skelton, J. M.; Frost, J. M.; Terban, M. W.; Zhai, H.; Alatas, A.; Owen, J. S.; Walsh, A.; Billinge, S. J. L. ACS Energy Lett. 2016, 1, 880.

(56) Leguy, A. M. A.; Goni, A. R.; Frost, J. M.; Skelton, J.; Brivio, F.; Rodriguez-Martinez, X.; Weber, O. J.; Pallipurath, A.; Alonso, M. I.; Campoy-Quiles, M.; Weller, M. T.; Nelson, J.; Walsh, A.; Barnes, P. R. F. Phys. Chem. Chem. Phys. 2016, 18, 27051.

(57) Frost, J. M.; Butler, K. T.; Brivio, F.; Hendon, C. H.; van Schilfgaarde, M.; Walsh, A. Nano Lett. 2014, 14, 2584.

(58) Laage, D.; Hynes, J. T. Science 2006, 311, 832.

(59) Fecko, C. J.; Loparo, J. J.; Roberts, S. T.; Tokmakoff, A. J. Chem. Phys. 2005, 122, 054506.

(60) Shinokita, K.; Cunha, A. V.; Jansen, T. L. C.; Pshenichnikov, M. S. J. Chem. Phys. 2015, 142, 212450.

(61) Weller, M. T.; Weber, O. J.; Henry, P. F.; Di Pumpo, A. M.; Hansen, T. C. Chem. Commun. 2015, 51, 4180.

(62) Lazonder, K.; Pshenichnikov, M. S.; Wiersma, D. A. Opt. Lett. 2006, 31, 3354.

(63) Sheng, C.; Zhang, C.; Zhai, Y.; Mielczarek, K.; Wang, W.; Ma, W.; Zakhidov, A.; Vardeny, Z. V. Phys. Rev. Lett. 2015, 114, 116601. 